\title{
THE FORMATION OF INFRARED RYDBERG LINES
}

\author{
ROBERT J. RUTTEN \\ Sterrekundig Instituut, Postbus $80000, N L-3508$ TA, Utrecht, The Netherlands \\ and \\ MATS CARLSSON \\ Institute of Theoretical Astrophysics, P.O. Box 1029, Blindern, N-0315 Oslo 3, Norway
}

\begin{abstract}
We review the formation of infrared solar spectral lines from highly excited levels in neutral atoms. The lines of $\mathrm{MgI}$ and $\mathrm{HI}$ are the most interesting ones. We explain the NLTE processes by which they are affected and we study the sensitivity of the $\mathrm{MgI} 12 \mu \mathrm{m}$ lines to granulation and to fluxtubes.
\end{abstract}

Key words: atomic processes - hydrogen - infrared: stars - line: formation - line: profiles magnesium - Sun: atmosphere

\section{Introduction}

In his concluding remarks to the Sixth Cambridge Cool Star workshop, Linsky (1990) listed the formation of the $\mathrm{MgI} 12 \mu \mathrm{m}$ emission features in the solar spectrum as one of the "major unanswered questions for the next workshop". By the next Cool Star workshop this enigma was indeed solved, by Carlsson et al. (1990, 1992a, 1992b) and Chang et al. (1991, 1992). In this review, we expand on our detailed analysis in Carlsson et al. (1992a, henceforth Paper I) and its Cool Star summary (Carlsson et al., 1992b) with further explanation of the Mg I $12 \mu \mathrm{m}$ emission, additional $\mathrm{MgI}$ infrared absorption line modeling, and a parallel discussion of $\mathrm{H} \mathrm{I}$ infrared line formation. The older literature is reviewed in Paper I. Relevant atomic physics is discussed by Chang (1993) in the preceding paper in this volume; in the next one, Avrett (1993) displays additional Mg I and H I computations. Our H I modeling is presented in Carlsson and Rutten (1992, henceforth Paper II) and elsewhere in this volume (Carlsson and Rutten, 1993).

All the above modeling is "one-dimensional", assuming lateral homogeneity. In view of the obvious inhomogeneity of the real solar photosphere, Linsky (1990) also put the apparent validity of such plane-parallel modeling for solar Fe I and other lines in the visible, as reviewed by Rutten (1990), on his list of major unanswered questions for the future, and suggested that things may be different in the infrared. We show below that the infrared transitions of $\mathrm{MgI}$, both the emission and the absorption lines, are actually better reproduced by our one-dimensional computations than any other Fraunhofer species sofar. We try to answer the question why this modeling is so good by computing Mg I $12 \mu \mathrm{m}$ profiles from granules.

More generally, using the infrared high- $\boldsymbol{n}$ lines to study the spatial structure of the solar atmosphere becomes possible now that their NLTE formation is basically understood. The strongest $\mathrm{HI}$ lines provide diagnostics of the chromosphere while the strongest $\mathrm{Mg}$ I lines are useful for upper-photosphere magnetometry. We lay a basis for the latter by computing Mg I $12 \mu \mathrm{m}$ profiles from magnetic fluxtubes. 


\section{NLTE Rydberg Line Formation}

\subsection{NLTE Populations}

Figure 1 shows the principal ingredients of Rydberg (high- $n$ ) line formation in the Sun. The lefthand panel is from Paper I and specifies LTE Rydberg populations $N^{*}$ for the MACKKL quiet-Sun model atmosphere (Maltby et al., 1986). It illustrates that infrared lines of neutral metals ( $\mathrm{Mg} \mathrm{I}, \mathrm{Si}$ I, Al I etc.) come from the upper photosphere, with Mg I supplying the strongest ones. In contrast, the stronger hydrogen lines have split formation. The lower chromosphere and lower photosphere both contribute opacity to them, with a wide gap at the temperature minimum (located at $\lg \tau_{500}=-4$ ). Such behavior is well known for $\mathrm{H} \alpha$ (e.g., Schoolman, 1972); it is shared by the higher HI $n \alpha$ lines (see the contribution functions in Carlsson and Rutten, 1993).
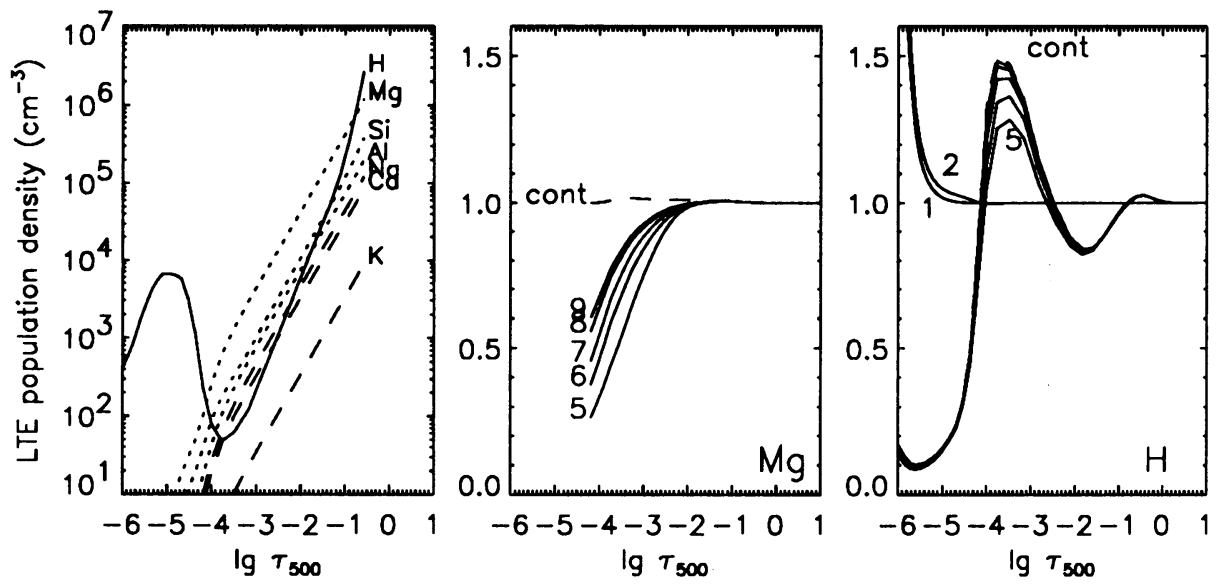

Fig. 1. Left:Saha-Boltzmann populations $N^{*}$ of comparable high- $n$ levels in various atoms. Middle and right: NLTE departure coefficients $\beta_{n}$ of levels $n=5-9$ and the ion for $\mathrm{MgI}$ and $\mathrm{HI}$, respectively; levels 1 and 2 added for H I. Abscissae: logarithm of continuum optical depth at $\lambda=500 \mathrm{~nm}$.

The curves in the lefthand panel shift upward for increasing $n$, but the pattern remains the same. Since the quantum defects of the neutral-atom levels decrease with increasing $n$, the corresponding Rydberg transitions (split in $l$ ) approach each other towards longer wavelengths and merge with the hydrogen lines from the short wavelength side. The $\mathrm{MgI}$ and $\mathrm{HI}$ lines dominate the resulting features in the spectrum. The MgI lines are stronger at $12 \mu \mathrm{m}$ (Brault and Noyes, 1983), whereas the 11-12 and 12-13 $\mathrm{HI}$ lines are about as strong as their $\mathrm{Mg}$ I counterparts. $\mathrm{HI}$ dominates at higher $n$, above $\lambda=100 \mu \mathrm{m}$ (Boreiko and Clark, 1986).

The other panels of Figure 1 are from Papers I and II, respectively. They show NLTE population departure coefficients $\beta_{n}=N / N^{*}$, with $N$ the actual NLTE population of level $n$. Note that these Zwaan coefficients $\beta_{n}$ differ from the more commonly used Menzel coefficients $b_{n}$. The latter are normalized by the $N / N^{*}$ ratio of the next ion and smaller by $1 / \beta_{\text {cont }}=N_{\text {cont }}^{*} / N_{\text {cont }}$ (Menzel and Cillié, 1937; 
Wijbenga and Zwaan, 1972; cf. Vernazza et al., 1981 p. 663). The LTE populations $N^{*}$ of $\mathrm{HI}$ and $\mathrm{MgI}$ in the lefthand panel should be multiplied by $\beta$ values from the middle and righthand panels to obtain actual MACKKL populations. The main change is a tenfold reduction of the chromospheric $\mathrm{H}$ I population peak. The patterns remain similar, however.

\subsection{NLTE MeChanisms}

The divergences between adjacent curves in the $\beta$ panels of Figure 1 cause NLTE emission in the corresponding infrared $\mathrm{MgI}$ and $\mathrm{HI}$ transitions. How do these departure divergences come about? We refer to Papers I and II for details; here, we illustrate the pertinent properties of high- $n$ levels more generally. These are the following:

(i)-Rydberg lines have long wavelengths. In the infrared, the correction factor $1-\left(\beta_{u} / \beta_{l}\right) \exp (-h \nu / k T)$ for stimulated emission reaches zero already for small excess of the upper-level departure $\beta_{u}$ over the lower-level departure $\beta_{l}$, i.e., for slight departure divergence. For example, for $T=5000 \mathrm{~K}$ and $\lambda=12 \mu \mathrm{m}, \exp (-h \nu / k T)=$ 0.79 , so that a departure ratio $\beta_{u} / \beta_{l}>1.27$ already produces a negative correction factor (lasering). For a smaller offset of $\beta_{u} / \beta_{l}$ from unity the line extinction decreases by the correction factor, while the line source function increases by its inverse. These changes differ qualitatively. The opacity decreases rapidly with height but the source function does not, especially in the upper photosphere (where the temperature gradient is small) and in the infrared (where the temperature sensitivity of the Planck function is slight). The effect of a small $\beta_{u}>\beta_{l}$ departure divergence is therefore much larger for the source function than for the opacity. Figure 11 of Paper I demonstrates that a departure excess of only $5 \%\left(\beta_{u} / \beta_{l}=1.05\right)$ already produces emission in the $12 \mu \mathrm{m}$ lines. Thus, as small as the departure divergences in Figure 1 are, their effects are large.

(ii)-Rydberg levels are hydrogenic. The fact that classical theory applies well to them not only facilitates model-atom building for NLTE computations, but also results in regular patterns in collision cross-sections. Figure 5 of Paper I shows the bound-bound collision matrix of our $\mathrm{Mg}$ I model atom. Close-lying levels with the same $n$ are coupled strongly through "l-changing" collisions with electrons and neutral hydrogen atoms. The resulting high-l same- $n$ manifolds are primarily coupled by $\Delta n=1$ collisions. Together, these constitute a "Rydberg ladder" along which collisional coupling is strongest.

(iii) - Rydberg transitions have small energy. Their energy separations are much smaller than the $1 \mathrm{eV}$ kinetic energy typically available in collisions. In addition, Rydberg atoms are very large. Therefore, collision rates dominate over radiative rates. The collisional coupling between Rydberg levels is very strong, as is their collisional coupling to the continuum. NLTE departure divergences can therefore only exist between Rydberg levels if they are driven by processes elsewhere in the Grotrian diagram.

Indeed, there are also non-Rydberg ingredients to the realization of Rydberg statistical equilibrium. These are:

(i) - NLTE photon pumping. Non-local photons that are sufficiently energetic to 


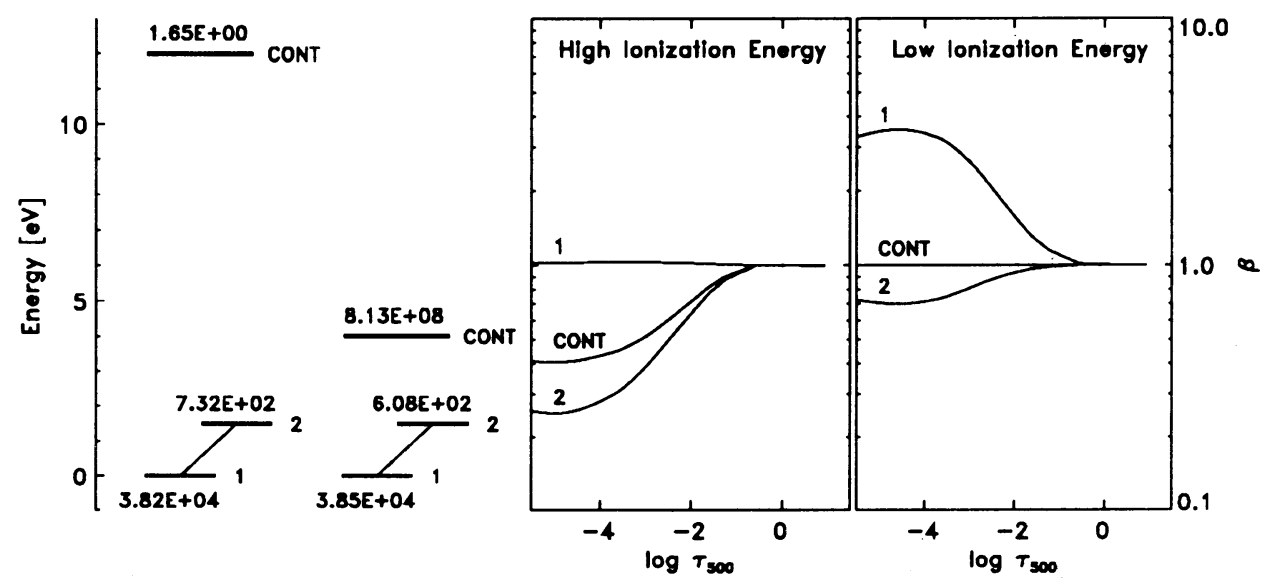

Fig. 2. Didactic computation of photon suction. Left: two-level-plus-continuum model atoms. The lefthand atom has $12 \mathrm{eV}$ ionization energy, the righthand atom $4 \mathrm{eV}$. The numbers specify NLTE population densities in $\mathrm{cm}^{-3}$ at $\log \tau_{500}=-3$. The abundances are scaled to obtain similar extinction in the single line, which resembles KI $769.9 \mathrm{~nm}$. In the lefthand case, the neutral stage contains most of the element; at right, the element is ionized. Right: corresponding NLTE departure coefficients against continuum optical depth. The split between $\beta_{1}$ and $\beta_{2}$ is set by two-level resonance scattering. Photon suction occurs for the case of low ionization energy at right; it produces overpopulation of the neutral stage $\left(\beta_{1}\right)$. Graph taken from Bruls (1992).

impart non-local information on local populations are either incoming or outgoing. In the first case, they cause optical pumping; in the second, photon suction. Photospheric photon pumping is best known in the form of ultraviolet overionization, for example of Fe I (Lites, 1972; Athay and Lites, 1972; $c f$. Rutten, 1988, 1990), but line pumping occurs also, for example in Fe II (Cram et al., 1980). Photon pumping employs ultraviolet radiation fields from the deeper photosphere, which are characterized by superthermal radiation temperatures because their mean intensity $J_{\nu}$ drops less steeply with height than the Planck function $B_{\nu}$. The corresponding excess in available photons causes larger upward than downward radiative rates and depletes the lower-state populations until these are small enough to attract a balancing downward net collisional rate.

(ii)-NLTE photon suction. This process follows from photon losses and operates in minority species such as the photospheric alkalis. It is demonstrated in Figure 2 after Bruls (1992), for two-level-plus-continuum model atoms following Bruls et al. (1992). Radiative deexcitations transfer electrons out of the upper level 2 into the lower level 1 . Loss of some of the resulting photons to outer space results in underpopulation of level 2 and overpopulation of level 1 . The resulting split between $\beta_{1}$ and $\beta_{2}$ starts deeply, due to strong resonance scattering; it produces the characteristic line source function deficit $S^{1}<B_{\nu}$ of a resonance line. In the case of high ionization energy (lefthand Grotrian diagram and lefthand $\beta$ panel), 
$\beta_{1}$ departs much less from unity than $\beta_{2}$ because the population of the ground state is much larger, as shown by the population densities in the Grotrian diagrams. However, $\beta_{1}$ rises well above unity in the case of low ionization energy; this overpopulation arises through bound-free coupling. The ion (CONT) is coupled to levels 1 and 2 by three-body recombination, with stronger coupling to level 2 than to level 1. In the lefthand case of large ionization energy, the ion has very small population and follows level 2 into depopulation. However, if the ion population is much larger than the neutral-atom population, it constrains the population of level 2 rather than be wagged around. Photon losses from level 2 are then compensated collisionally from the ion. This is photon suction. It increases the population of the ground state until the neutral-stage overpopulation $\left(\beta_{1}\right)$ is sufficiently large to balance the ionization equilibrium with a net collision rate up from level 1 . Whereas the photon-loss divergence between $\beta_{1}$ and $\beta_{2}$ is the same for the two cases, producing similar NLTE $S^{1}<B_{\nu}$ source function behavior, the photon suction produces triple overpopulation of the neutral stage in the case of low ionization energy. Bruls et al. (1992) supply further demonstrations of the suction process using realistic K I model atoms of increasing size.

(iii) - LTE reservoirs. For low ionization energy, the ion population acts as a large reservoir which is impervious to the neutral atom and its photon losses. It is an LTE reservoir, with $\beta_{\text {cont }} \approx 1$, because it contains most of the species. For high ionization energy, the ground state of the neutral atom constitutes the LTE reservoir. In that case, the continuum population follows the photon losses.

(iv) - NLTE replenishment flows. In the case of low ionization energy, the photon losses and the continuum reservoir supply boundary conditions to the establishment of net recombination into level 2. This constitutes a replenishment flow, driven by the resonance line. In real atoms, intermediate levels at the bottom of the Rydberg ladder and the continuum at the top supply similar boundary conditions. Departure divergence between these is then accommodated along the ladder, preferentially in small $\Delta n=1$ steps. A radiative-collisional departure diffusion flow results. It is similar to the Rydberg population diffusion occurring in optically-thin recombination (e.g., Seaton, 1964; Section 5.4 of Sobelman et al., 1981; Biberman et al., 1987). For sufficiently high levels, it is fully dominated by collisions, with $\Delta n=1$ collisions carrying most of the net downward flow.

\subsection{NLTE in Mg I INFRAREd LiNeS}

Figure 3 has a schematic representation of the $\mathrm{MgI}$ term diagram at left. Magnesium is predominantly singly ionized in the photosphere, so that the $\mathrm{Mg}$ II ground state constitutes the $\mathrm{Mg}$ LTE population reservoir. The lowest $\mathrm{Mg}$ I levels are too far from the continuum for ultraviolet overionization, which is most effective at $3-4 \mathrm{eV}$ ionization energies $(\lambda=400-300 \mathrm{~nm})$. The resonance transitions and other strong lines such as $3 \mathrm{~s}-3 \mathrm{p}$ and the $b$ lines are opaque and close to detailed balance. The main NLTE driver consists of a sequence of $\Delta n=1$ and $\Delta n=2$ lines at high $l$. These are strong in the laboratory sense, having large transition probabilities. The lines are sufficiently weak in the solar spectrum, due to their small Boltzmann populations, that they are thin in the middle photosphere. Their combined radiative 
losses cause photon suction, replenishing photon losses from the continuum just as in the neutral alkalis. The resulting overpopulation of levels 3 (comparable to level 1 in the two-level case, split in $l$ ) and the superthermal radiation in their bound-free continua balance the ionization equilibrium.

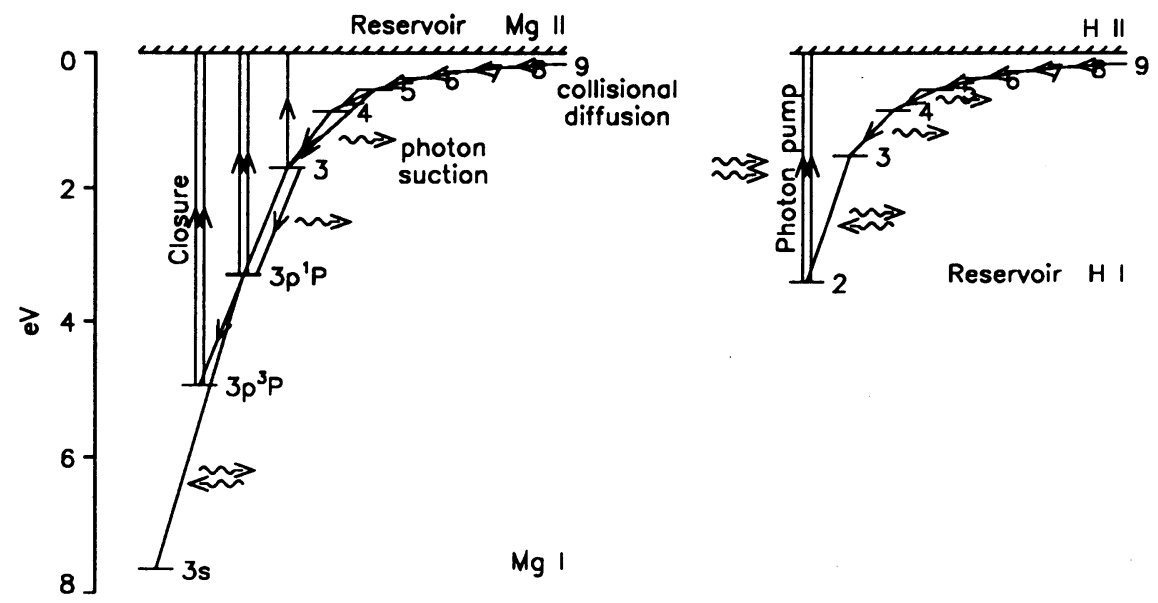

Fig. 3. Schematic Grotrian diagrams of MgI (left) and H I (right), with the continua aligned. In Mg I, the NLTE driving is primarily by photon losses in infrared lines with large transition probability. These are replenished from the LTE continuum reservoir along a Rydberg ladder of $\Delta n=1$ steps because the latter levels have very strong collisional coupling. In $\mathrm{HI}$, the lower levels constitute the reservoir and the Balmer continuum is the main NLTE driver in the temperature minimum region. It pumps the continuum into overpopulation and so produces departure diffusion along the Rydberg ladder.

The replenishment flow comes down primarily along the Rydberg ladder. It is collisionally dominated along its top. This is visualized in Figure 4. The lefthand waterfalls illustrate that most of the replenishment flow enters at the top of our model $(n=9)$. The $\mathrm{Mg}$ II -9 waterfall would undoubtedly be split into yet higher steps if our atomic model accommodated these, but such splitting would not affect the 7-6 step which harbors the $12 \mu \mathrm{m}$ lines. The cascade at right shows the split between net collisional and radiative deexcitation along $\Delta n=1$ steps. Collisions already dominate the 7-6 transition, and gain even more higher up.

In Paper I and in Carlsson et al. (1992b) we have shown results of various experiments and of a "multi-MULTI" sensitivity analysis. In the latter the code (named MULTI; Carlsson, 1986) was run 2525 times, doubling each input parameter one by one. The results demonstrate that the NLTE driving is indeed by infrared photon losses with some help from violet overionization, that the replenishment flow takes $\Delta n=1$ ladder steps preferentially, and that the flow is made up by collisions along this ladder. Therefore, the $12 \mu \mathrm{m}$ lines have NLTE emission peaks by virtue of the strong collisional coupling along the ladder steps. Only if this coupling is strong 

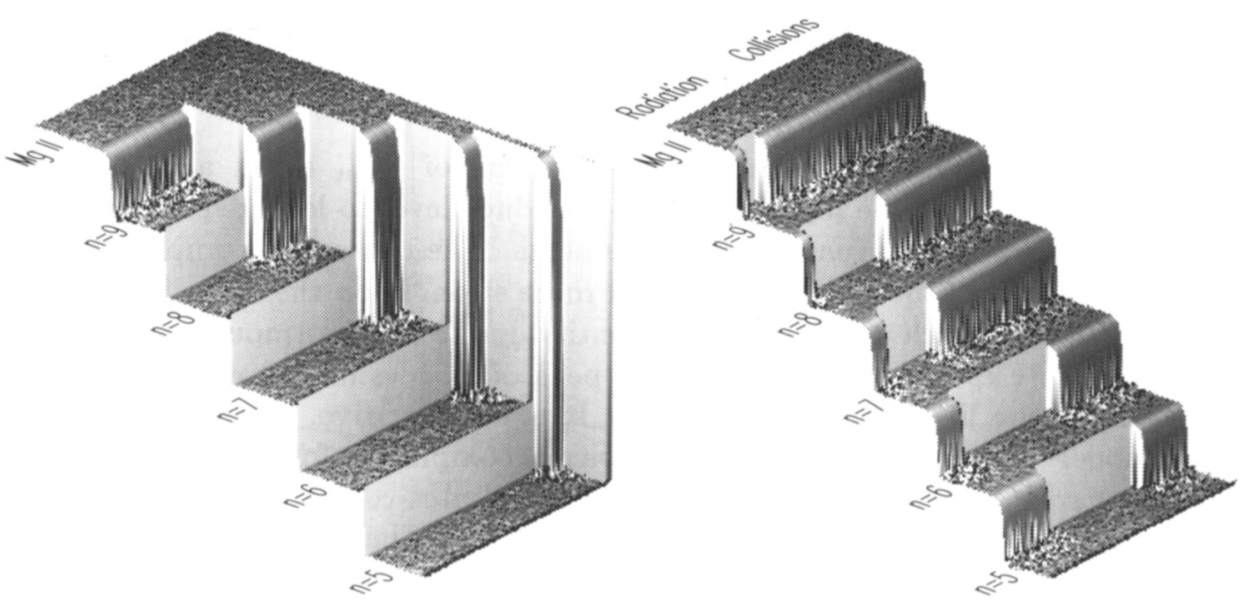

Fig. 4. Kayaker's views of the population departure diffusion flow along the Rydberg ladder in $\mathrm{Mg}$ I. Left: distribution of the total net recombination over levels 5-9. Right: division between collisions and radiation of the net downward rates along $\Delta n=1$ steps.

enough does the replenishment flow come down along the laddder, rather than using other channels such as $\Delta n=2$ level skipping or direct recombination. The step-by-step adjustments to the boundary conditions along the ladder maintain level-to-level departure divergences. The $\mathrm{MgI} 12 \mu \mathrm{m}$ lines have NLTE emission peaks because of strong collisional coupling between the pertinent levels!

In addition, the sensitivity experiments show that our computation is very robust. The largest changes to the computed $12 \mu \mathrm{m}$ peaks amount to only $20 \%$ when doubling transition probabilities (bound-bound or bound-free), $10 \%$ for doubling collision cross-sections.

\subsection{NLTE IN H I INFRARED LINES}

Hydrogen is neutral in the photosphere. Levels 1 and 2 (with Ly $\alpha$ in detailed balance) constitute the LTE reservoir. The Balmer continuum has $3.4 \mathrm{eV}$ ionization energy and feeds strongly on superthermal radiation with $J_{\nu}>B_{\nu}$ from the deep photosphere. This pumping is a major NLTE driver. Photon losses in the principal Paschen and Bracket transitions probably contribute as well.

The resulting $\mathrm{HI} \beta$ pattern in Figure 1 differs from the $\mathrm{Mg}$ I pattern primarily in that the ion population is out of LTE. The initial hump and dip of $\beta_{\text {cont }}$ stem from reversed $B_{\nu}>J_{\nu}$ excesses in infrared continua and photon exchange between infrared lines and their background continua, while the peak in the temperature minimum region is caused by Balmer continuum pumping. The wide minimum at large height results from the chromospheric temperature rise.

Note that the H I $\beta$ panel differs from the corresponding Menzel $b$ departure 
plot in Figure 30 of Vernazza et al. (1981), which has reversed peaks and dips and $b \approx 1$ for high- $n$ levels in the low chromosphere. Note also that much of the H I recombination literature (references in paper II) adopted $\beta_{\text {cont }}=1$ implicitly. This assumption is invalid in the low chromosphere where hydrogen is predominantly neutral and $\beta_{\text {cont }} \approx 0.1$.

In the temperature minimum region, the peak of $\beta_{\text {cont }}$ again acts as upper boundary condition to the high- $n$ ladder to produce level-to-level departure divergence and NLTE source function enhancements. These are less computationally robust than for $\mathrm{MgI}$, because they depend more strongly on the $J_{\nu}>B_{\nu}$ excesses in the ultraviolet which are difficult to quantify due to the enormous line crowding in that region. Experiments show that the peak of $\beta_{\text {cont }}$ is indeed very sensitive to the details of the line haze representation. It is not sensitive, however, to change of the model atom size, an experiment easier accomplished for $\mathrm{HI}$ than for $\mathrm{MgI}$. We computed $\mathrm{HI}$ lines with $n<10$ for an 11-level-plus-continuum model in addition to the 19-level-plus-continuum model of Paper II. There is no difference in the resulting profiles.

\subsection{Mg I Rydberg Line Profiles}

Figure 5 is assembled from Paper $I$ and shows our results for the strongest infrared emission line, $\mathrm{Mg}$ I $12.32 \mu \mathrm{m}$. The fit is quite good. Note that we applied no parameter fitting in our computation. Standard choices were made for microturbulence $\left(1.0 \mathrm{~km} \mathrm{~s}^{-1}\right)$, macroturbulence $\left(1.5 \mathrm{~km} \mathrm{~s}^{-1}\right)$, and van der Waals damping enhancement (times 2.5). Other choices were made regarding neutral hydrogen collisions (assumed sufficiently important in l-changing transitions to produce detailed balancing of close-lying levels with equal $n-c f$. Omont, 1977) and for forbidden transitions (smaller collision strengths than comparable permitted transitions). As noted above, the resulting line profiles are not very sensitive to the cross-sections. What counts is the regularity of the patterns in the collision matrix, and the corresponding selection of the Rydberg ladder as primary replenishment channel.

The righthand panel of Figure 5 shows the computed source function with superimposed formation heights. Comparison with the lefthand panel illustrates the mapping of source function structure into line profile. The peaks originate in the upper photosphere even when observed near the limb.

The modeling of Chang et al. (1991) reproduces the observed $12 \mu \mathrm{m}$ profiles less well than ours. Chang et al. (1992) added computations of other infrared $\mathrm{Mg}$ I lines, observed by ATMOS (Farmer and Norton, 1989) and examined by Jefferies (1991). Their new computations also do not reproduce the observations very closely. At Avrett's request, we have computed the $7 g-6 f$ and $6 h-5 g$ lines using the setup of Paper I without change, except that $l$-splitting was taken into account by adopting detailed balance between the corresponding fine structure levels. Figure 6 shows results (actually computed during the meeting, and kindly displayed by Avrett in his talk). These fits are good too. It appears that we have a sound rendering of solar Mg I atoms inside our computer. We suspect that the major difference from Chang et al. lies in collision matrices and the treatment of photoionization. The atom-size experiment with $\mathrm{HI}$ indicates that our $\mathrm{MgI}$ atom (complete to $n=9$ ) is 

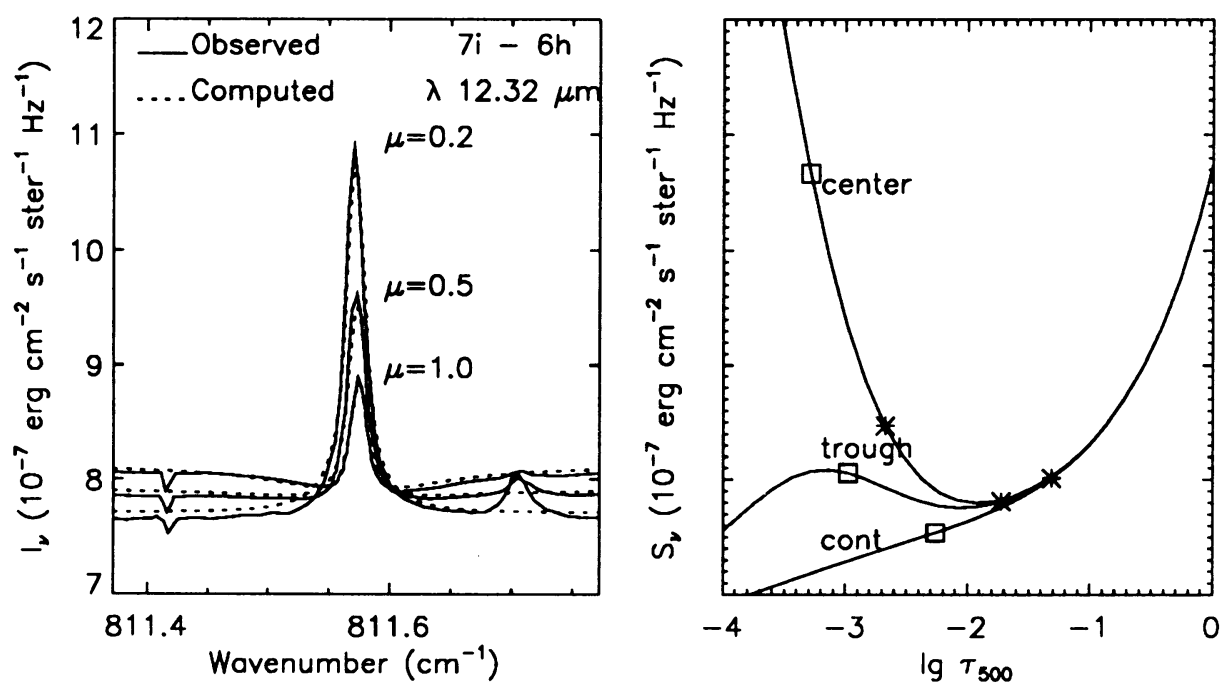

Fig. 5. Profiles and source functions of the $\mathrm{MgI} 12.32 \mu \mathrm{m}$ line $(7 i-6 h)$. Left: observed and computed profiles on absolute intensity scales, for the three viewing angles $\theta$ indicated by $\mu=\cos \theta$. Observed profiles are based on Brault and Noyes (1983). Right: computed source functions, for line center, an inner-wing wavelength ("trough") and the continuum. Average formation heights are specified by squares for $\mu=0.2$, stars for $\mu=1$.
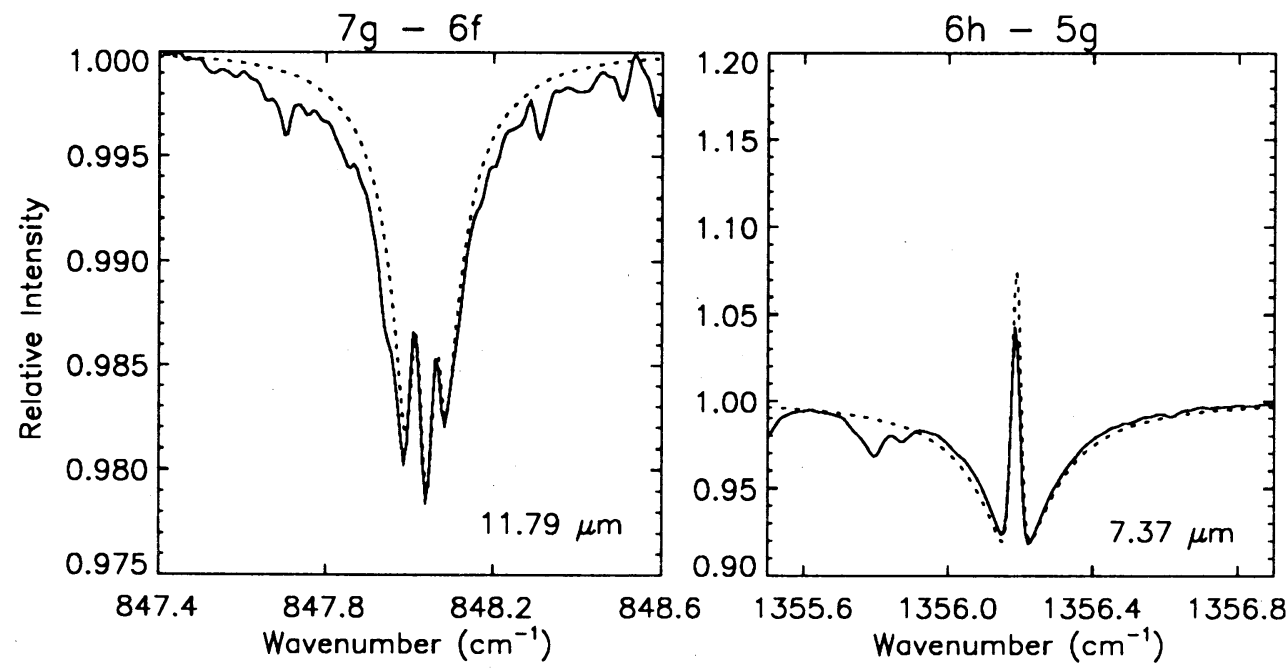

Fig. 6. Modeling (dashed) of two Mg I absorption lines using the setup of Paper I. Observed profiles (solid) are from ATMOS. 
sufficiently large, as does the waterfall visualization in Figure 4.

There are also differences between the $\mathrm{HI}$ profiles displayed in our contribution elsewhere in this volume and Avrett's H I profiles in the next paper. These are likely to arise from different line-haze formalisms which affect the Balmer continuum (opacity distribition functions versus opacity sampling functions, though both from Kurucz's line tables; LTE versus scattering).

\section{The Real Sun: Granules}

The good fits which our MgI computations produce without any parameter adjustment rather seem too good, in the sense that plane-parallel modeling has never managed to reproduce center-to-limb profile behavior of multiple lines in any spectral species without resorting to adjustment of the many fitting parameters represented by height and angle-dependent micro- and macro-turbulence and damping enhancements. The most successful example to date is the analysis of the $\mathrm{NaI}$ spectrum by Caccin et al. (1980), which indeed relied on such fitting.
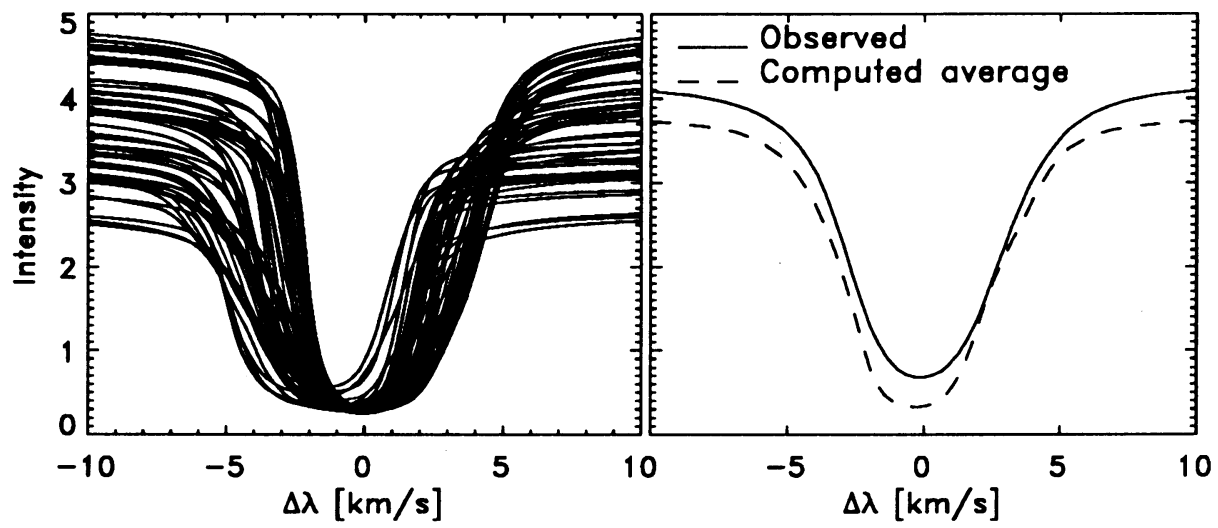

Fig. 7. Profiles of K I $769.9 \mathrm{~nm}$ computed from a numerical simulation of the solar granulation by Nordlund and Stein, after Bruls and Rutten (1992). Left: profiles per resolved surface element. Right: spatially averaged profiles. Dashed: average of the computed profiles. Solid: observed. Abscissae: wavelength expressed as Dopplershift, with upward velocity (blueshift) positive. Ordinates: absolute intensity in $10^{-5} \mathrm{erg} \mathrm{s}^{-1} \mathrm{~cm}^{-2} \mathrm{~Hz}^{-1} \mathrm{ster}^{-1}$.

Why is our Mg I modeling so good? We suspect that the success of these planeparallel computations of infrared Rydberg lines is enhanced by lessened response to photospheric inhomogeneities. In a parallel analysis, Bruls and Rutten (1992) computed alkali line profiles using output from a Nordlund \& Stein granulation simulation as input (cf. Stein and Nordlund, 1989; Nordlund and Stein, 1990). Results are shown in Figure 7. The lefthand panel shows profiles of the K I $769.9 \mathrm{~nm}$ resonance line per simulation surface element. No turbulent broadening or damping enhancement was employed. The granulation produces appreciable broadening of the mean profile (righthand panel), which will be hard to match precisely with one-dimensional micro- and macroturbulence or collisional damping enhancements. 


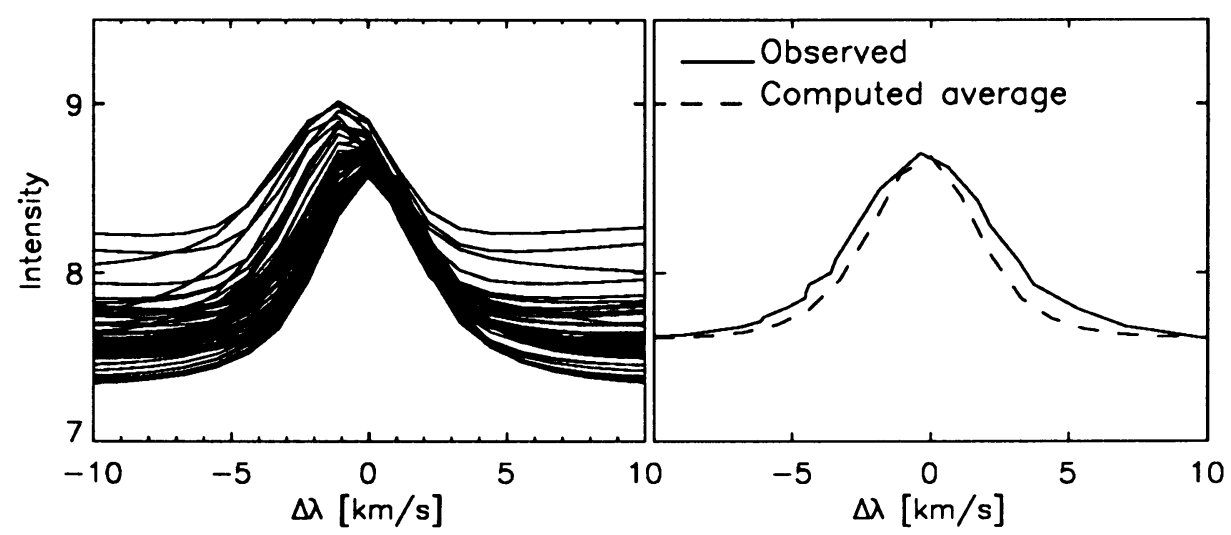

Fig. 8. Same as Figure 7, but for the $\mathrm{Mg} \mathrm{I} 12.32 \mu \mathrm{m}$ line. Ordinates: absolute intensity in $10^{-7} \mathrm{erg} \mathrm{s}^{-1} \mathrm{~cm}^{-2} \mathrm{~Hz}^{-1} \mathrm{ster}^{-1}$. Note the offset from zero.

The $\mathrm{Mg}$ I $12.32 \mu \mathrm{m}$ line originates from the upper photosphere, as does the core of $\mathrm{KI} 769.9 \mathrm{~nm}$. Figure 8 shows results for the $\mathrm{MgI}$ line from a similar experiment, using the same Nordlund \& Stein simulation data. Again, there is much variation in the left panel whereas the computed average is close to the observed profile. However, there are significant differences between the two tests. The K I background continuum originates much deeper in the photosphere and therefore displays much larger granular modulation, up to $50 \%$. The granular contrast in the $\mathrm{MgI}$ background continuum ranges over less than $10 \%$. In $\mathrm{KI}$, the granular correlation between upward motion and large continuum brightness produces distinct asymmetry in the resolved profiles, both per profile and in their occurrence patterns. The resolved MgI profiles display more symmetry and less or even reversed Dopplershift-brightness correlation. Note that these profiles are presented on velocity scales to make them comparable. The line cores have similar widths (about $10 \mathrm{~km} \mathrm{~s}^{-1}$ at their base) but the $\mathrm{MgI}$ wings, which map source function structure, are more extended. In summary, the Mg I $12 \mu \mathrm{m}$ line suffers much less from granulation-induced asymmetries than the $\mathrm{K} I$ resonance line because its background continuum originates higher. Gaussian broadening, as prescribed by microand macro-turbulence, may therefore indeed work better as simulacrum.

\section{The Real Sun: Fluxtubes}

The MgI $12 \mu \mathrm{m}$ emission features are promising magnetometers, although their Landé factors $g_{\text {eff }}$ are only unity. The widths of their emission peaks, set by the NLTE source function structure, are reasonably small (Figure 8). This NLTE sharpness and their clean hydrogen-like splitting pattern gives the $\mathrm{Mg}$ I peaks their value. On the other hand, their NLTE origin requires Detailed line formation modeling for any application beyond simple splitting measurement. We display initial NLTE magnetic-profile computations here.

Figure 9 shows profiles and contribution functions for the $12.32 \mu \mathrm{m}$ line. We 

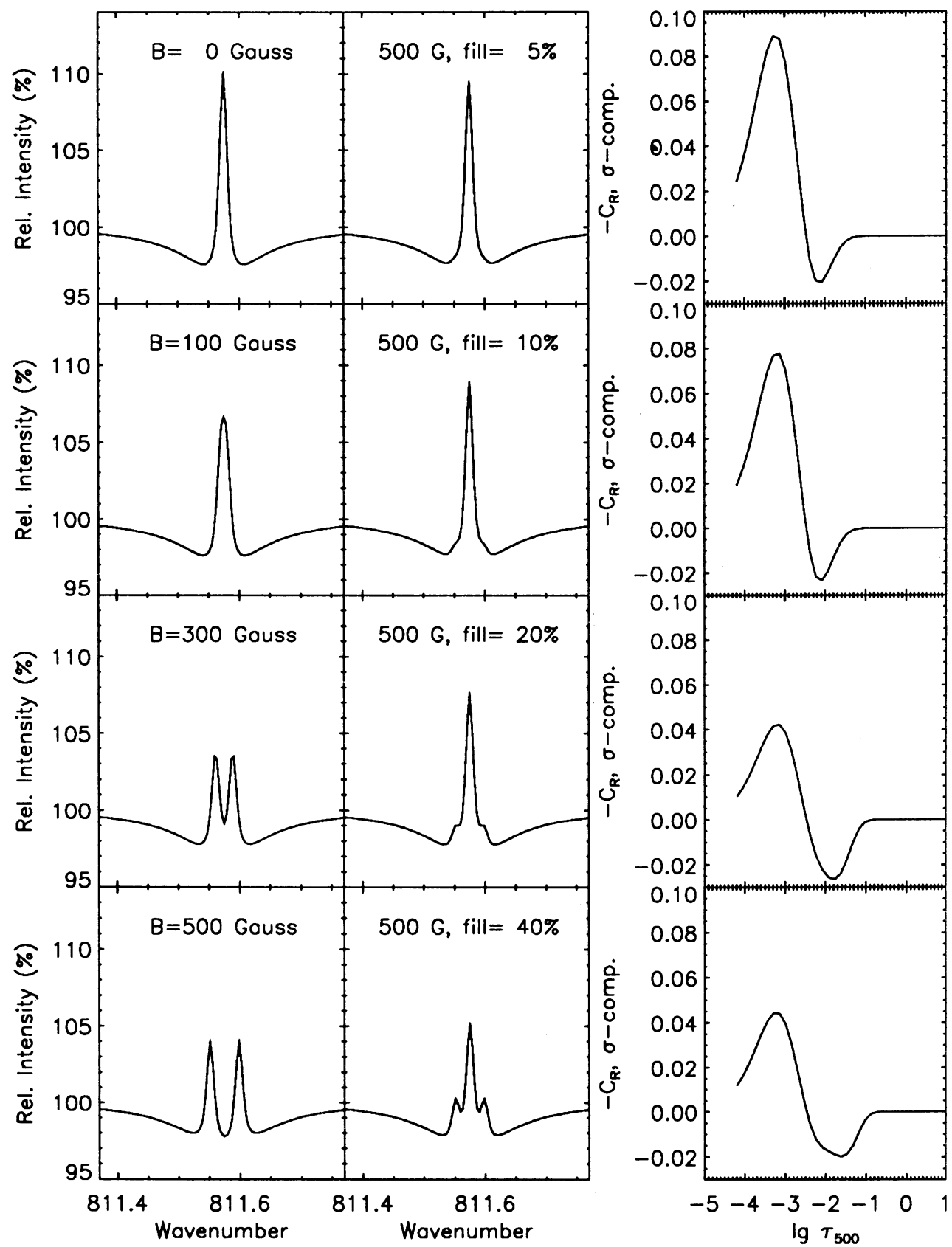

Fig. 9. Magnetic-field modeling of the $\mathrm{MgI} 12.32 \mu \mathrm{m}$ line. Left: fluxtube profiles for the indicated field strengths. Middle: non-magnetic profiles mixed with $500 \mathrm{G}$ profiles to the specified filling factors. Right: $\sigma$-component contribution functions, corresponding to the field strengths in the lefthand column. 
have adopted the field-free approximation (Rees, 1969, 1987), $g_{\text {eff }}=1$, the T5780 model atmosphere (Paper I), and height-independent vertical fields of the specified strengths. The lefthand column shows intensity profiles from such first approximations to a solar fluxtube. The $\sigma$ components are well separated above $B=300 \mathrm{G}$. The middle column shows profiles computed for a mix of $500 \mathrm{G}$ and non-magnetic plasma specified by the filling factor. The filling factor must be appreciable to deliver noticeable $\sigma$ components. The quiet Sun does not contain sufficient field to upset our plane-parallel reproduction of its spatially-averaged profiles.

Finally, the righthand column displays relative-intensity contribution functions to a $\sigma$ component, corresponding to the field strengths in the lefthand column. These are line-depression contribution functions as defined by Magain (1986), plotted negatively to let emission be contributed by an upward peak. They are similar to the non-magnetic line center contribution function plotted in Figure 7 of Paper I, at half the magnitude for a fully split profile.

\section{Conclusion}

The previous reviews of infrared line formation were the Liège ones of de Jager (1964, 1975). In 1964, he remarked that for $\lambda>2 \mu \mathrm{m}$ the solar spectrum was disappointingly void of spectral lines, none having been identified above $\lambda=5 \mu \mathrm{m}$. In 1975 he had to quote his 1964 review, because nothing had changed.

In 1992, we at last have at least infrared Mg I and $\mathrm{H} \mathrm{I}$ lines of interest. Neither species is valuable for granulation research. High-resolution narrow-band images taken in $\mathrm{Mg}$ I $12.32 \mu \mathrm{m}$ will be remarkably bland, except for regions of close-packed strong magnetic fields (Figures 8 and 9). This confirms that the primary $\mathrm{MgI}$ interest lies in upper-photosphere plage and penumbra magnetometry. The $\mathrm{H} \mathrm{I}$ lines appear useful as diagnostics of the temperature and density structure of the low chromosphere (Paper II - see also our poster contribution and Avrett's review in this volume).

Using $\mathrm{MgI}$ and $\mathrm{HI}$ emission features to measure properties of the solar atmosphere requires detailed non-LTE modeling. Complete model atoms are then needed, as well as proper accounting for Stark broadening and Stark shifts and correct treatment of the ultraviolet line haze. These tasks are not trivial.

\section{References}

Avrett, E. H.: 1993, in these proceedings.

Athay, R. G. and Lites, B. W.: 1972, Astrophys. J. 176, 809.

Biberman, L. M., Vorob'ev, V. S., and Yakubov, I. T.: 1987, Kinetics of Nonequilibrium LowTemperature Plasma, Plenum, New York.

Boreiko, R. T. and Clark, T. A.: 1986, Astron. Astrophys. 157, 353.

Brault, J. and Noyes, R.: 1983, Astrophys. J. 269, L61.

Bruls, J. H. M. J.: 1992, Formation of diagnostic lines in the solar spectrum, PhD Thesis, Utrecht University.

Bruls, J. H. M. J. and Rutten, R. J.: 1992, Astron. Astrophys. 265, 257.

Bruls, J. H. M. J., Rutten, R. J., and Shchukina, N. G.: 1992, Astron. Astrophys. $265,237$.

Caccin, B., Gomez, M. T., and Roberti, G.: 1980, Astron. Astrophys. 92, 63. 
Carlsson, M.: 1986, A Computer Program for Solving Multi-Level Non-LTE Radiative Transfer Problems in Moving or Static Atmospheres, Report No. 33, Uppsala Astronomical Observatory.

Carlsson, M. and Rutten, R. J.: 1992, Astron. Astrophys. 259, L53 (Paper II).

Carlsson, M. and Rutten, R. J.: 1993, in these proceedings.

Carlsson, M., Rutten, R. J., and Shchukina, N. G.: 1990, in L. Deszö (Ed.), The Dynamic Sun, Proc. EPS $6^{\text {th }}$ European Solar Meeting, Publ. Debrecen Heliophysical Observatory 7, Debrecen, p. 260.

Carlsson, M., Rutten, R. J., and Shchukina, N. G.: 1992a, Astron. Astrophys. 253, 567 (Paper I).

Carlsson, M., Rutten, R. J., and Shchukina, N. G.: 1992b, in M. S. Giampapa and J. A. Bookbinder (Eds.), Cool Stars, Stellar Systems, and the Sun, Proc. Seventh Cambridge Workshop, ASP Conf. Series, 26, p. 518.

Chang, E. S.: 1993, in these proceedings.

Chang, E. S., Avrett, E. H., Mauas, P. J., Noyes, R. W., and Loeser, R.: 1991, Astrophys. J. 379, L79.

Chang, E. S., Avrett, E. H., Mauas, P. J., Noyes, R. W., and Loeser, R.: 1992, in M. S. Giampapa and J. A. Bookbinder (Eds.), Cool Stars, Stellar Systems, and the Sun, Proc. Seventh Cambridge Workshop, ASP Conf. Series, 26, p. 521.

Cram, L. E., Rutten, R. J., and Lites, B. W.: 1980, Astrophys. J. 241, 374.

de Jager, C.: 1964, in M. Migeotte (Ed.), Les Spectres Infrarouges des Astres, Coll. Int. Liège, Mém. $8^{\circ}$ Soc. Roy. Sci., Liège, p. 151.

de Jager, C.: 1975, Space Sci. Rev. 17, 645.

Farmer, C. B. and Norton, R. H.: 1989, A High-Resolution Atlas of the Infrared Spectrum of the Sun and the Earth Atmosphere from Space, NASA Ref. Publ. 1224, Vol. 1.

Jefferies, J. T.: 1991, Astrophys. J. 377, 337.

Linsky, J. L.: 1990, in G. Wallerstein (Ed.), Cool Stars, Stellar Systems and the Sun, Proc. Sixth Cambridge Workshop, Astron. Soc. Pac. Conf. Series 9, p. 500.

Lites, B. W.: 1972, Observation and Analysis of the Solar Neutral Iron Spectrum, NCAR Cooperative Thesis No. 28, High Altitude Observatory, Boulder.

Magain, P.: 1986, Astron. Astrophys. 163, 135.

Maltby, P., Avrett, E. H., Carlsson, M., Kjeldseth-Moe, O., Kurucz, R. L., and Loeser, R.: 1986, Astrophys. J. 306, 284.

Menzel, D. H. and Cillié, G.: 1937, Astrophys. J. 85, 88.

Nordlund, $A$. and Stein, R. F.: 1990, Comp. Phys. Comm. 59, 119.

Omont, A.: 1977, Journal de Physique 38, 1343.

Rees, D. E.: 1969, Solar Phys. 10, 268.

Rees, D. E.: 1987, in W. Kalkofen (Ed.), Numerical Radiative Transfer, Cambridge University Press, Cambridge, Great Britain, p. 213.

Rutten, R. J.: 1988, in R. Viotti, A. Vittone, and M. Friedjung (Eds.), Physics of Formation of FeII Lines Outside LTE, IAU Colloquium 94, Reidel, Dordrecht, p. 185.

Rutten, R. J.: 1990, in G. Wallerstein (Ed.), Cool Stars, Stellar Systems and the Sun, Proc. Sixth Cambridge Workshop, Astron. Soc. Pac. Conf. Series, Volume 9, p. 91.

Schoolman, S. A.: 1972, Solar Phys. 22, 344.

Seaton, M. J.: 1964, Mon. Not. R. Astron. Soc. 127, 177.

Sobelman, I. I., Vainshtein, L. A., and Yukov, E. A.: 1981, Excitation of Atoms and Broadening of Specral Lines, Springer, Berlin.

Stein, R. F. and Nordlund, Å.: 1989, Astrophys. J. Lett. 342, L95.

Vernazza, J. E., Avrett, E. H., and Loeser, R.: 1981, Astrophys. J. Suppl. Ser. 45, 635.

Wijbenga, J. W. and Zwaan, C.: 1972, Solar Phys. 23, 265. 